\title{
Necrotising retinopathies simulating acute retinal necrosis syndrome
}

\author{
B Balansard, B Bodaghi, N Cassoux, C Fardeau, S Romand, F Rozenberg, N A Rao, P LeHoang
}

See end of article for authors' affiliations

.....................

Correspondence to: $P$ LeHoang, $M D, P h D$, Department of Ophthalmology, PitiéSalpêtrière Hospital, 43 bd de l'Hôpital, Paris, France; bahram.bodaghi@ psl.ap-hop-paris.fr

Accepted for publication 4 June 2004
Br J Ophthalmol 2005;89:96-101. doi: 10.1136/bjo.2004.042226 simulate acute retinal necrosis (ARN).

Methods: Retrospective non-comparative case series. The charts of 16 patients presenting with a clinical impression of ARN at Pitié-Salpêtrière Hospital, Paris, France, between 1994 and 1999, who required initial antiviral therapy were reviewed. All of the patients had extensive laboratory tests. Anterior chamber paracentesis was performed on 14 patients and evaluated by polymerase chain reaction (PCR) and/or the Witmer-Goldmann coefficient to determine the cause of retinitis. Three of the 14 cases also had diagnostic vitrectomy. Responses to specific treatment, initiated based on laboratory results, and the final outcome were evaluated.

Results: Seven of the 16 patients were female and nine were male. The retinitis was bilateral in five patients and unilateral in 11 patients. The average age of the patients at presentation was 53.6 years. 13 patients were immune deficient for various reasons. Upon initial presentation, the patients' visual acuities were less than $20 / 200$ in $68 \%$ of the affected eyes. The final diagnoses, based on laboratory data and therapeutic response were toxoplasmic retinochoroiditis $(62.5 \%)$, syphilitic retinitis $(12.5 \%)$, aspergillus endophthalmitis (12.5\%), Behçet's disease (6.2\%), and intraocular lymphoma (6.2\%). Visual acuity was stabilised or improved in 12 patients (75\%). Two patients with aspergillosis died despite antifungal therapy.

Conclusions: Toxoplasmic retinochoroiditis is the major cause of retinal necrosis that simulates ARN, and PCR analysis of the aqueous humour is helpful in establishing the diagnosis. Such atypical toxoplasma retinochoroiditis may be associated with poor visual outcome. is immunocompetent patients, acute retinal necrosis (ARN) is characterised by peripheral retinal necrosis and is associated with retinal arteritis, prominent vitritis, and inflammatory reaction in the anterior chamber. ${ }^{1}$ This syndrome, first described in 1971, is mainly caused by either varicella zoster virus (VZV) or herpes simplex virus (HSV). Rare cases are caused by cytomegalovirus (CMV). ${ }^{12}$ The diagnosis of ARN is usually based on clinical findings and the response to an antiviral agent such as aciclovir.

Despite the clinical criteria proposed by Holland et al in 1994, a diagnosis of ARN may be difficult. Clinical presentations range from focal to extensive retinal necrosis and the clinical course ranges from mild to fulminating. ${ }^{1-5}$ Moreover, in some patients the clinical findings can be atypical and may not be clear enough to make a definitive diagnosis or to promptly initiate an antiviral agent. The delay in diagnosis can lead to loss of vision that could otherwise be prevented. In addition, similar necrotising retinitis may also occur from non-viral infectious agents including Toxoplasma gondii, bacteria, or fungi. ${ }^{6-10}$ Other disease entities (namely, retinal vasculitis, intraocular tumours, and sarcoidosis) can also present with retinitis that mimics ARN. ${ }^{11-20}$ Thus, specific and sensitive laboratory tests are necessary to confirm a diagnosis of ARN or the non-viral retinopathies that present with features simulating ARN.

The aim of this study is to detect various causes of necrotising retinitis which present with clinical features that simulate ARN.

\section{PATIENTS AND METHODS}

In this retrospective study, we reviewed the charts of 16 patients who were examined between 1994 and 1999 at PitiéSalpêtrière Hospital, Paris, France. The initial clinical diagnosis for all 16 patients was ARN; all were subsequently found to have non-viral necrotising retinitis. All 16 patients presented with anterior uveitis, vitritis, and/or retinal vasculitis associated with well demarcated areas of mid-retinal or peripheral confluent retinal infiltrates and a clinical aspect of necrosis (fig 1). Lesions were usually large, multifocal, diffuse, and unilateral. Because of its potential severity and the importance of early antiviral therapy, the diagnosis of necrotising viral retinopathy was initially suspected in all cases.

An anterior chamber paracentesis was performed in 14 patients (table 1), and three patients also underwent a diagnostic vitrectomy rapidly after referral. Intraocular lymphoma was suspected in one case and two patients presented with retinal detachment complicating retinal necrosis. The vitrectomy sample for patient 15 was submitted for cytology. A volume of $150 \mu \mathrm{l}$ of aqueous humour $(\mathrm{AH})$ was aspirated using a 30 gauge needle that passed through the limbus; $500 \mu \mathrm{l}$ of undiluted vitreous were also obtained during the vitrectomy. Informed consent was obtained from all patients. The polymerase chain reaction (PCR) analysis of the AH and vitreous for the herpesviruses and for Toxoplasma gondii were conducted concomitantly with the determination of the Witmer-Goldmann coefficient in paired AH and serum samples. The test results were interpreted by a masked observer who was unaware of the clinical history or the findings of the patient. In addition, serology tests for syphilis, a tailored laboratory examination and a systemic evaluation were performed on all patients. All patients initially received systemic antiviral agents. A diagnosis of Behçet's disease was

Abbreviations: $A H$, aqueous humour; $A R N$, acute retinal necrosis: CMV, cytomegalovirus; EBV, Epstein-Barr virus; HSV, herpes simplex virus; PCR, polymerase chain reaction; VZV, varicella zoster virus 


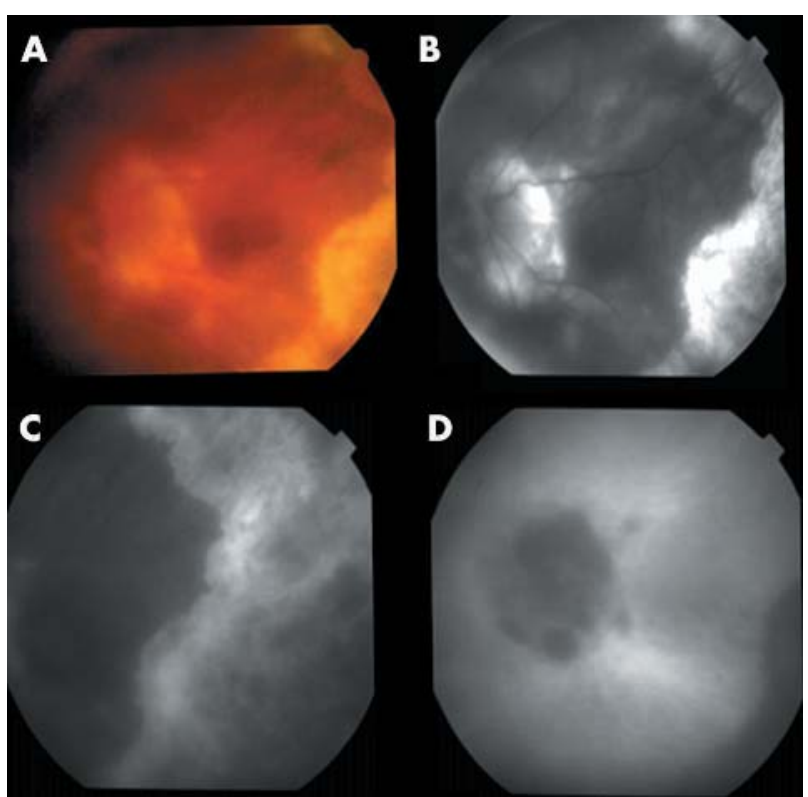

Figure 1 Toxoplasma peripheral retinitis. (A) Fundus photography, (B, C) Fluorescein angiography; (D) ICG angiography. Note necrosis involving peripheral retina.

considered when the patients met the criteria of the International Study Group for Behçet's disease. ${ }^{21}$

\section{PCR analysis}

A volume of 10-20 $\mu \mathrm{l}$ of AH was used for each PCR reaction. DNA was obtained by either phenol:chloroform extraction or ethanol precipitation. A single PCR for HSV-1, HSV-2, and Epstein-Barr virus (EBV) was performed as previously described. ${ }^{22}$ The amplified products were detected on ethidium stained agarose gels, then characterised by their specific migration pattern before and after digestion with restriction enzymes SmaI and BamHI. The sensitivity of this technique was evaluated at $5 \times 10^{2}$ genome equivalents $(\mathrm{GEq}) / \mathrm{ml}$ for HSV- 1 and $2 \times 10^{3} \mathrm{GEq} / \mathrm{ml}$ for HSV-2 by a European quality control HSV test panel. Specific CMV and VZV-PCR assays were performed using published procedures. ${ }^{23-25}$ Using the same technique, 2-20 copies of EBV-DNA could be detected, as previously described. The sensitivity of the published CMV and VZV-PCR tests was evaluated by a serial dilution of infected cell culture supernatants. The results were $10^{-6}$ for VZV and $10^{-5}$ for CMV plaque forming units. The amplified products were detected on agarose gels, and their specificity was confirmed by hybridisation with digoxigenin labelled specific oligonucleotide probes.

A previously described technique was used to detect $T$ gondii. ${ }^{26}$ Briefly, the primers used for amplification were B22 5' AACGGGCGAGTAGCACCTGAGGAGA 3' (sense) and B23 5' TGGGTCTACGTCGATGGCATGACAAC 3' (antisense). The presence of inhibitors within the samples was detected by internal control within reaction mixtures. The sensitivity of the PCR assay was monitored using a control sample that contained a single parasite.

\section{Intraocular anti-T gondii antibody production}

The total amount of IgG amount and specific anti-T gondii IgG titre was determined for each serum and AH sample. ${ }^{26}$ The total IgG concentration was measured by nephelometry, and specific anti-T gondii titres were assessed by the use of a high sensitivity agglutination test. The Witmer-Goldmann coefficient $(\mathrm{C})$ was calculated by the following formula: $\mathrm{C}=\mathrm{Cl} / \mathrm{C} 2$, where $\mathrm{Cl}=$ anti- $T$ gondii IgG titre/total IgG concentration within ocular fluid, and C2 represents the same ratio within serum. A Witmer-Goldmann coefficient of 3.0 or greater is considered proof of antibody production within the ocular tissue, thus indicating ocular toxoplasmosis. ${ }^{26}$

\section{RESULTS}

Of the total of 16 cases reviewed, seven of the patients were women and nine were men. Their ages ranged from 20 to 85 years (mean 53.6 years). Five of the patients presented with bilateral process. Thirteen of the 16 patients had immune suppression; nine of these were taking systemic corticosteroids or cytotoxic agents; five had haematological abnormalities; and three were HIV positive (table 1). Seven of the 16 patients $(43.8 \%)$ had a medical history of mucocutaneous or systemic viral infection; there were four cases of HSV, two cases of VZV, and one case of CMV.

The ophthalmological findings are summarised in table 1. Acute retinal necrosis was initially suspected in all patients. All 16 patients presented with at least two criteria for the diagnosis of ARN as defined by the American Uveitis Society, ${ }^{3}$ and all received antiviral medication: eight received aciclovir, six received foscarnet, and two received ganciclovir.

Viral serologies were performed for each patient, and all of them were seropositive for VZV, HSV, or CMV. Serology for toxoplasmosis was positive in 13 of the cases. The AH-PCR for $T$ gondii DNA was positive in eight cases (table 1). However, of the three PCR tests performed for patient 7 , the first two were negative, but the third revealed $T$ gondii amplified products. Two AH samples taken from patient 10 were both negative, and the final diagnosis of toxoplasmosis was based on an adequate therapeutic response to antitoxoplasma agents. The Witmer-Goldmann coefficient was positive (greater than 3.0) in three cases (patients 5, 8, and 9) (table 1). Patients 5 and 8 had elevated coefficients (greater than 3.0) for toxoplasmosis in both the $\mathrm{AH}$ and vitreous. Herpesviridae DNA analysis was negative in all cases, except for patient 7. VZV-DNA was found in the AH of this patient, but he did not respond to the antiviral agent; 125 days later, a third AH-PCR analysis was positive for T gondii. We could not determine whether the first test result for VZV was a false positive, or if the patient had another infection along with the ocular toxoplasmosis. However, this patient responded to the anti-toxoplasma agents, pyrimethamine and sulfadiazine.

Syphilitic retinitis was diagnosed in two patients after cerebrospinal fluid and blood serologies (Treponema pallidum haemaglutination and VDRL were found positive) (patients 11 and 12) and both responded to penicillin treatment (see fig 2). Ocular lymphoma was diagnosed in a patient after cytological examination of the vitreous specimen was performed (patient 15). Behçet's disease was subsequently diagnosed in a patient (patient 16) based on the International Diagnostic Criteria. The patient's antiviral drugs were discontinued after the results of an anterior chamber paracentesis for herpesviruses were negative. The patient's intraocular inflammation responded positively to high doses of corticosteroids. The PCR results for the above four patients were negative for herpesviruses and for $T$ gondii. Final diagnosis of aspergillus retinitis was made for two patients (13 and 14) based on subsequent systemic evidence for aspergillosis and the findings of a cerebral MRI.

The patients with a positive PCR and/or the WitmerGoldmann coefficient for $T$ gondii (table 1 ) and patient 10 received pyrimethamine and sulfadiazine; the latter drug was replaced by clindamycin in one patient because of an allergic reaction. All of the patients with laboratory evidence of $T$ gondii also received systemic corticosteroids.

The two patients with syphilitic retinitis were treated with intravenous penicillin for a 3 week period. One patient died (patient 13) from aspergillus septicaemia before treatment 


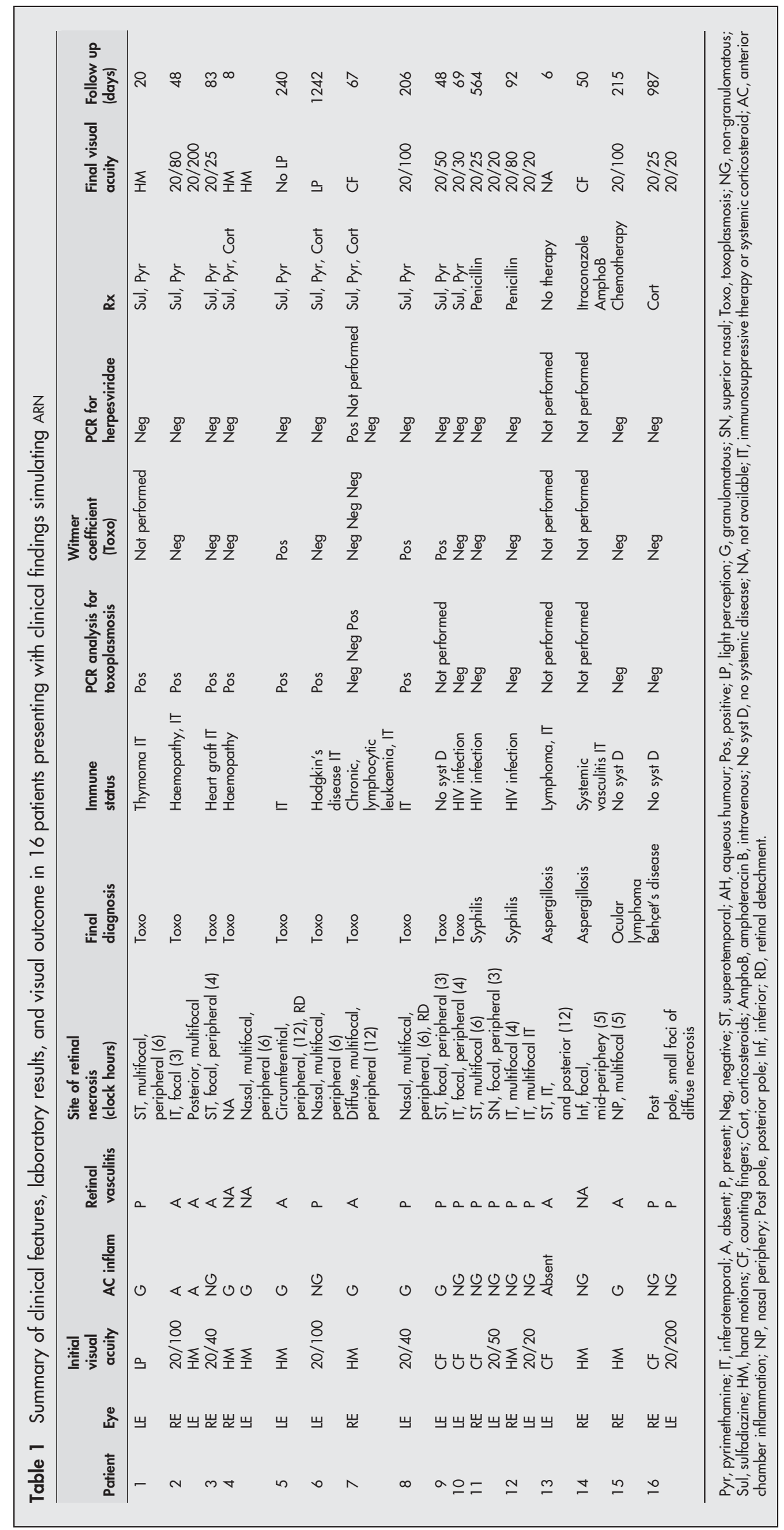




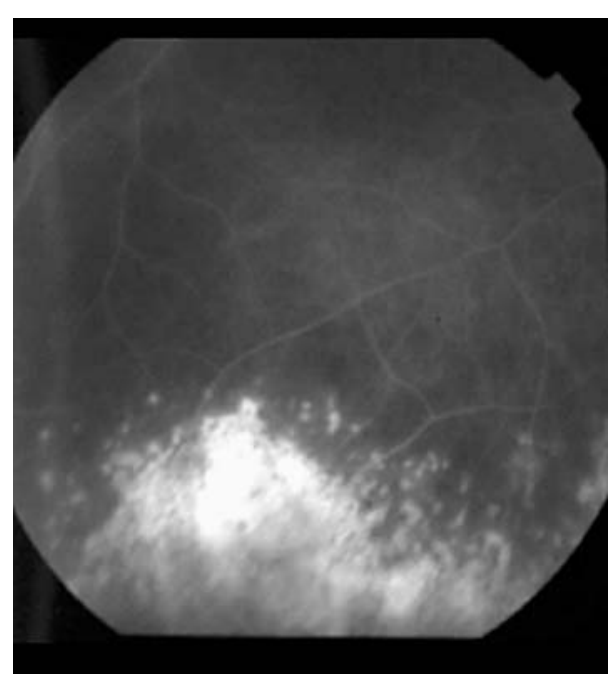

Figure 2 Syphilitic retinitis. Intermediate phase of fluorescein angiogram showing small hyperfluorescent foci of retinal necrosis near a peripheral confluent zone.

for the aspergillus retinitis could be initiated. A postmortem examination confirmed the diagnosis of aspergillosis. The second patient with culture proved aspergillosis received intravenous itraconazole and intravitreal amphotericin B, but died a few days later from the systemic infection. The treatment of the other cases and final visual acuities are summarised in table 1 . Visual acuity was stabilised or improved in 12 patients $(75 \%)$, and it was improved by more than two lines on the Snellen chart in six patients (37.5\%). However, the final visual outcome was worse in those cases with toxoplasmic retinochoroiditis (table 1).

The time between the discovery of initial symptoms and a final diagnosis (table 1) ranged from 3 days to 296 days (mean 106 days). In addition to ocular toxoplasmosis $(62.5 \%)$, there were other causes of retinitis including ocular lymphoma $(6.2 \%)$, Behçet's disease $(6.2 \%)$, syphilitic retinitis $(12.5 \%)$, and aspergillus endophthalmitis (12.5\%).

\section{DISCUSSION}

Necrotising retinopathies represent a diagnostic and therapeutic challenge. In such cases, ARN is usually suspected, since it carries a poor visual outcome and a high rate of complications, such as rhegmatogenous retinal detachment. However, as noted in this study, there are other infectious and non-infectious causes that can present with clinical features suggestive of ARN that may also carry a poor prognosis. These simulating conditions include peripheral toxoplasma retinitis, syphilis, Behçet's disease, intraocular lymphoma and aspergillosis. Since a vast majority of the patients have positive serologies for herpesviruses and for $T$ gondii, such laboratory investigations may not be helpful in reaching proper aetiological diagnosis. However, serology for syphilis proved to be useful in this series of patients.

Paracentesis is a safe procedure that allows for PCR analysis of $\mathrm{AH}$ for the detection of infectious agents. ${ }^{27}$ The procedure is useful in the diagnosis of ARN and progressive outer retinal necrosis in both immunocompromised and immunocompetent patients. ${ }^{19-32}$ It may also confirm a clinical diagnosis of herpetic infection, with a sensitivity ranging from $50 \%$ to $100 \%$. We have recently reported a series of 22 patients (29 eyes) who presented with necrotising herpetic retinitis, and AH-PCR reliably detected the pathogenic agent in $86.4 \%$ of the cases. ${ }^{22}$ Patients with necrotising herpetic retinopathies were younger (mean age: 44.1 years) than those reported in our present series (mean age
53.6 years). Compared with atypical toxoplasmosis, viral retinopathies have a dramatically rapid evolution. In the latter, the whole peripheral retina may be involved within a few days or a few weeks leading to retinal necrosis, retinal detachment, and permanent visual loss. Therefore, the mean duration before diagnostic confirmation was 4.5 weeks in the group with viral retinopathy and 8.5 weeks in the group with atypical toxoplasmosis. Aspergillosis may have the same type of evolution but death occurs rapidly as a result of systemic infection and despite specific therapy. The evolution of ocular lymphoma is similar to that of atypical toxoplasmosis. Based on our previous studies, it seems that the diagnosis of necrotising viral retinopathy may be overestimated in 30\% of cases before molecular diagnosis. Current and previous studies of highly sensitive and specific PCR based assays may contribute to both the diagnosis and the management of patients with necrotising retinitis. The benefit of a test reliable enough to produce a rapid, accurate diagnosis and lead to appropriate treatment may outweigh the theoretical risks of the procedure.

It has been reported that PCR analysis of both aqueous and vitreous were equivalent measures for the detection of viral DNA in patients presenting with ARN. ${ }^{28}$ False positive results are exceptional. ${ }^{19}{ }^{33}$ Knox et al and Garweg and Bohnke studied the usefulness of herpesvirus DNA identification in atypical cases of retinal necrosis. ${ }^{19}{ }^{34}$ They confirmed both the sensitivity and specificity of this method: no more than one herpesvirus genome was found in viral retinitis, and there were no false negative results for toxoplasmosis, syphilis, Behçet's disease, or idiopathic retinitis. However, false positive results have been reported in two patients with endophthalmitis who had positive VZV nucleic acid sequences and two other cases of toxoplasmic retinochoroiditis that revealed amplified CMV-DNA. ${ }^{32}{ }^{33}$ In our series, the PCR for patient 7 initially indicated the presence of VZV nucleic acid amplified products, but the patient was subsequently diagnosed with toxoplasmic retinochoroiditis based on therapeutic response; repeated tests confirmed toxoplasma DNA amplified products in the $\mathrm{AH}$.

A Witmer-Goldmann coefficient determination for the AH may also be useful in the diagnosis of toxoplasmic retinochoroiditis. $^{35-38}$ According to Davis et al, a simultaneous coefficient determination for both $T$ gondii and the herpesviruses is essential for the interpretation of positive results for necrotising retinitis, and they consider a coefficient of more than one to be significant. ${ }^{39}$ Davis et al also found a $78 \%$ sensitivity and a $90 \%$ specificity in the diagnosis of ARN by Witmer-Goldmann coefficient determination. However, false negative results have been reported in immunocompromised patients; de Boer reported two immunocompromised patients who did not produce local antibodies, but whose aqueous samples contained $T$ gondii DNA. ${ }^{27}$ In our series, PCR analysis for $T$ gondii seems to be a more sensitive indicator than does the coefficient determination in immunocompromised patients. Patients 1, 2, 3, 4, and 6 had no local antibody production, even though the PCR analysis was positive. Alternatively, patient 9 was immunocompetent, but also had a positive coefficient determination. These results are similar to our previous report on the diagnosis of toxoplasma retinitis in an immunocompromised host. ${ }^{26}$

Montoya found a $100 \%$ sensitivity and specificity for $T$ gondii DNA in the vitreous fluid of patients with ocular toxoplasmosis. ${ }^{40}$ However, de Boer et al, Bou et al, and Aouizerate et al highlighted the lack of sensitivity of this method. ${ }^{27}{ }^{41}{ }^{42}$ Similarly, in a series of 31 healthy patients with a clinical diagnosis of ocular toxoplasmosis, Garweg and Bohnke found only one positive PCR result out of $43 \mathrm{AH}$ samples that were tested..$^{34}$ In immunocompetent individuals, the lack of sensitivity of this method may be alleviated by 
employing the Witmer-Goldmann coefficient determination. $^{36}{ }^{42}$ In our present series, the coefficient was the same in both the $\mathrm{AH}$ and vitreous. Although we did not compare the aqueous and vitreous humour antibody coefficients, our study showed other causes for the necrotising retinitis in both immunocompetent and immunodeficient patients (table 1). Davis et al considered both $\mathrm{AH}$ and vitreous to be equivalent determinants of local antibody production. ${ }^{39}$

Atypical toxoplasmic retinochoroiditis continues to be the primary differential diagnosis for ARN, as revealed in this study. More than $62 \%$ of the cases that were initially diagnosed as ARN were subsequently re-evaluated as toxoplasma retinitis. Our results are similar to others who also named toxoplasmosis as the primary differential diagnosis for ARN in immunocompromised individuals. ${ }^{40}{ }^{43-49}$ However the present study has also revealed other causes for the necrotising retinitis, both in immunocompetent and immunodeficient patients (table 1).

In the present study, all 16 patients complained of having had blurred vision for several weeks (mean 3 months) and they all presented with anterior uveitis and vitritis. All 16 patients also had retinal necrosis without retinochoroidal scars. Taken together, these findings led to the diagnosis of ARN. The typical presentation of ocular toxoplasmosis includes focal retinitis at the border of a pre-existing pigmented retinochoroidal scar. Such typical manifestations may not be observed in all cases. However, there are some clinical features that can help to differentiate between ocular toxoplasmosis and ARN. In our series, the majority of patients presented with a large focus or multiple smaller foci of peripheral necrotising retinitis without adjacent pigmented retinal scars. Other features of disease may help to distinguish viral infections from parasitic retinitis. In the latter, the yellow-white aspect of lesions is denser, borders have a distinct, smooth contoured edge, retinal haemorrhages are less frequent, and vasculitis is located near necrotic foci. According to Johnson et al toxoplasmosis is less likely to involve the peripheral retina than ARN, or to include thumbprint patches at the border that lies between the necrosis and the normal retina..$^{45}$ Additionally, toxoplasmosis may reveal the presence of some uninvolved retina between the necrosis and the ora serrata. In the present study, these clinical features could not be appreciated because all of them had significant amounts of vitritis. Thus, PCR analysis was helpful for the diagnosis of toxoplasmosis and for differentiating between it and viral retinitis, fungal infections, or lymphoma. The present study also revealed the need for an extended treatment period (mean 15.6 weeks) in patients with toxoplasmosis. These cases may also have a poor visual prognosis (table 1 ).

In this series, ARN was initially suspected at the clinic evaluations; therefore, the patients received the antiviral therapy. The present study also demonstrates the importance of performing the proper diagnostic procedures to confirm aetiology. Disease progression despite the use of antiviral drugs, may be associated with a resistant form of viral retinitis, and would require a more aggressive therapy; but there is also a distinct possibility of the presence of other non-viral infections or of a malignant condition. When either a viral infection or ocular toxoplasmosis are suspected, $\mathrm{AH}$ analysis by PCR and the use of the Witmer-Goldmann coefficient appear to be the best methods for a final diagnostic confirmation. Results may also be obtained within 72 hours. Both antiparasitic and antiviral regimens may be required until a final diagnosis is obtained. Antiviral therapy was discontinued as soon as molecular diagnosis was confirmed. An average of 7 days was necessary in our series from ocular sampling until final diagnosis, leading to discontinuation of antiviral drugs. However, detection of specific intraocular antibody production may be negative if paracentesis is performed too early during the course of infection. This is usually not observed in long lasting, extensive $T$ gondii infection of the retina, in immunocompetent hosts. However, PCR will be more informative in immunocompromised patients. Molecular diagnosis of ocular toxoplasmosis is the major and earliest criteria for discontinuation of antivirals. The efficacy of antiparasitic drugs is an important, but secondary condition, as it may be evaluated only after a few weeks.

In conclusion, our study reveals that in addition to atypical toxoplasmic retinochoroiditis, there are several other disease entities that may mimic ARN; and the most common of these appears to be peripheral toxoplasma retinitis. Serology may not be helpful in differentiating between toxoplasmosis and viral retinitis. Aqueous humour analysis by PCR and a Witmer-Goldmann coefficient determination, along with other diagnostic proceedings are helpful for a proper diagnosis and for disease management. Similar to ARN, toxoplasma retinochoroiditis may also carry a poor prognosis in immunocompromised individuals.

\section{Authors' affiliations}

B Balansard, B Bodaghi, N Cassoux, C Fardeau, P LeHoang,

Department of Ophthalmology, Pitié-Salpêtrière Hospital, Paris, France S Romand, Institut de Puériculture, Paris, France

F Rozenberg, Laboratoire de Virologie, Hôpital Saint-Vincent de Paul, Paris, France

N A Rao, Doheny Eye Institute and the Department of Ophthalmology, Keck School of Medicine, University of Southern California, Los Angeles, CA, USA

The authors have no commercial interest in this work.

\section{REFERENCES}

1 Altamirano D, Rochat C, Claeys $M$, et al. Acute retinal necrosis: a result of immune dysfunction? Report of a case with subacute evolution and relapses in a patient with impaired cellular immunity. Ophthalmologica 1994;208:49-53.

2 Urayama A. Unilateral acute uveitis with retinal periarteritis and detachment. Jpn J Clin Ophthalmol 1971;25:607-19.

3 Holland GN. Standard diagnostic criteria for the acute retinal necrosis syndrome. Executive Committee of the American Uveitis Society. Am J Ophthalmol 1994;117:663-7.

4 Cunningham ET Jr, Short GA, Irvine AR, et al. Acquired immunodeficiency syndrome-associated herpes simplex virus retinitis. Clinical description and use of a polymerase chain reaction-based assay as a diagnostic tool. Arch Ophthalmol 1996;114:834-40.

5 Batisse D, Eliaszewicz M, Zazoun L, et al. Acute retinal necrosis in the course of AIDS: study of 26 cases. AIDS 1996; 10:55-60.

6 Dodds EM, Lowder CY, Boskovich SA, et al. Simultaneous syphilitic necrotizing retinitis and placoid chorioretinitis in acquired immune deficiency syndrome. Retina 1995; 15:354-6.

7 Shalaby IA, Dunn JP, Semba RD, et al. Syphilitic uveitis in human immunodeficiency virus-infected patients. Arch Ophthalmol 1997; 115:469-73.

8 Mendelsohn AD, Jampol LM. Syphilitic retinitis. A cause of necrotizing retinitis. Retina 1984;4:221-4.

9 Bouisse V, Cochereau-Massin I, Jobin D, et al. [Syphilitic uveitis and human immunodeficiency virus infection]. J Fr Ophtalmol 1991;14:605-9.

10 Cubillan LD, Cubillan EA, Berger TG, et al. Syphilitic uveitis and dermatitis. Arch Ophthalmol 1998;116:696-7.

11 de Smet MD, Nussenblatt RB, Davis JL, et al. Large cell lymphoma masquerading as a viral retinitis. Int Ophthalmol 1990;14:413-7.

12 Gass JD, Trattler HL. Retinal artery obstruction and atheromas associated with non-Hodgkin's large cell lymphoma (reticulum cell sarcoma). Arch Ophthalmol 1991;109:1134-9.

13 Ridley ME, McDonald HR, Sternberg P Jr, et al. Retinal manifestations of ocular lymphoma (reticulum cell sarcoma). Ophthalmology 1992;99:1153-60; discussion 1160-1.

14 Anteby I, Kramer M, Rahav G, et al. Necrotizing choroiditis-retinitis as presenting symptom of disseminated aspergillosis after lung transplantation. Eur J Ophthalmol 1997;7:294-6.

15 Bodoia RD, Kinyoun JL, Lou QL, et al. Aspergillus necrotizing retinitis. A clinico-pathologic study and review. Retina 1989;9:226-31.

16 Graham DA, Kinyoun JL, George DP. Endogenous Aspergillus endophthalmitis after lung transplantation. Am J Ophthalmol 1995; 1 19:107-9 
17 Weishaar PD, Flynn HW Jr, Murray TG, et al. Endogenous Aspergillus endophthalmitis. Clinical features and treatment outcomes. Ophthalmology 1998; 105:57-65.

18 Glacet-Bernard A, Laliam-Gheraieb L, Coscas G. [Viral vasculitis and disseminated white spots]. J Fr Ophtalmol 1994;17:281-5.

19 Knox CM, Chandler D, Short GA, et al. Polymerase chain reaction-based assays of vitreous samples for the diagnosis of viral retinitis. Use in diagnostic dilemmas. Ophthalmology 1998;105:3744; discussion 44-5.

20 Freilich JM, Ryan EA, Lou PL, et al. Acute retinal necrosis syndrome: findings, management, and differential diagnosis. Int Ophthalmol Clin 1996;36:141-6.

21 International Study Group for Behçet's Disease. Criteria for the diagnosis of Behçet's disease. Lancet 1990;335:1078-80.

22 Tran TH, Rozenberg F, Cassoux N, et al. Polymerase chain reaction analysis of aqueous humour samples in necrotising retinitis. $\mathrm{Br} J$ Ophthalmol 2003;87:79-83.

23 Lionnet $F$, Pulik M, Genet $P$, et al. Myelitis due to varicella-zoster virus in two patients with AIDS: successful treatment with acyclovir. Clin Infect Dis 1996:22:138-40.

24 Gozlan J, Salord JM, Roullet E, et al. Rapid detection of cytomegalovirus DNA in cerebrospinal fluid of AIDS patients with neurologic disorders. J Infect Dis 1992;166:1416-21

25 Puchhammer-Stockl E, Popow-Kraupp T, Heinz FX, et al. Detection of varicella-zoster virus DNA by polymerase chain reaction in the cerebrospinal fluid of patients suffering from neurological complications associated with chicken pox or herpes zoster. J Clin Microbiol 1991;29:1513-16.

26 Fardeau C, Romand S, Rao NA, et al. Diagnosis of toxoplasmic retinochoroiditis with atypical clinical features. Am J Ophthalmol 2002; 134:196-203.

27 De Boer JH, Verhagen C, Bruinenberg M, et al. Serologic and polymerase chain reaction analysis of intraocular fluids in the diagnosis of infectious uveitis. Am J Ophthalmol 1996;121:650-8.

28 Abe T, Tsuchida K, Tamai M. A comparative study of the polymerase chain reaction and local antibody production in acute retinal necrosis syndrome and cytomegalovirus retinitis. Graefes Arch Clin Exp Ophthalmol 1996;234:419-24.

29 Ganatra JB, Chandler D, Santos C, et al. Viral causes of the acute retinal necrosis syndrome. Am J Ophthalmol 2000;129:166-72.

30 Yamamoto S, Pavan-Langston D, Kinoshita S, et al. Detecting herpesvirus DNA in uveitis using the polymerase chain reaction. $\mathrm{Br} J$ Ophthalmol 1996;80:465-8.

31 Itoh N, Matsumura N, Ogi A, et al. High prevalence of herpes simplex virus type 2 in acute retinal necrosis syndrome associated with herpes simplex virus in Japan. Am J Ophthalmol 2000;129:404-5.

32 Verbraak FD, Galema M, van den Horn GH, et al. Serological and polymerase chain reaction-based analysis of aqueous humour samples in patients with AIDS and necrotizing retinitis. AIDS 1996;10:1091-9.
33 Short GA, Margolis TP, Kuppermann BD, et al. A polymerase chain reaction-based assay for diagnosing varicella-zoster virus retinitis in patients with acquired immunodeficiency syndrome. Am J Ophthalmol 1997:123:157-64

34 Garweg J, Bohnke M. Varicella-zoster virus is strongly associated with atypical necrotizing herpetic retinopathies. Clin Infect Dis 1997;24:603-8.

35 Garweg JG, Jacquier P, Fluckiger F. [Current limits in diagnosis of ocular toxoplasmosis]. Klin Monatsbl Augenheilkd 1998;212:330-3.

36 Bornand JE, de Gottrau P. Uveitis: is ocular toxoplasmosis only a clinical diagnosis? Ophthalmologica 1997;211:87-9.

37 Baarsma GS, Luyendijk L, Kijlstra A, et al. Analysis of local antibody production in the vitreous humor of patients with severe uveitis. Am J Ophthalmol 1991;112:147-50.

38 Haut J, Roman S, Morin Y, et al. [Search for etiology in 110 cases of uveitis. Value of punctures of the aqueous humor and vitreous body]. J Fr Ophtalmol 1995; 18:292-304.

39 Davis JL, Feuer W, Culbertson WW, et al. Interpretation of intraocular and serum antibody levels in necrotizing retinitis. Retina 1995;15:233-40.

40 Montoya JG, Parmley S, Liesenfeld O, et al. Use of the polymerase chain reaction for diagnosis of ocular toxoplasmosis. Ophthalmology 1999; 106:1554-63.

41 Bou G, Figueroa MS, Marti-Belda P, et al. Value of PCR for detection of Toxoplasma gondii in aqueous humor and blood samples from immunocompetent patients with ocular toxoplasmosis. J Clin Microbiol 1999;37:3465-8.

42 Aouizerate F, Cazenave J, Poirier L, et al. Detection of Toxoplasma gondii in aqueous humour by the polymerase chain reaction. $\mathrm{Br} J$ Ophthalmol 1993;77:107-9.

43 Manners RM, O'Connell S, Guy EC, et al. Use of the polymerase chain reaction in the diagnosis of acquired ocular toxoplasmosis in an immunocompetent adult. Br J Ophthalmol 1994;78:583-4.

44 Minihan M, Cleary PE, Cryan B, et al. Diagnosis of an atypical case of ocular toxoplasmosis using the demonstration of intraocular antibody production and the polymerase chain reaction. Br J Ophthalmol 1999;83:753-4.

45 Johnson MW, Greven GM, Jaffe GJ, et al. Atypical, severe toxoplasmic retinochoroiditis in elderly patients. Ophthalmology 1997; 104:48-57.

46 Benson MT, Parsons MA, Talbot JF, et al. Aggressive toxoplasma retinitis. Acta Ophthalmol (Copenh) 1992;70:795-800

47 Bosch-Driessen LH, Karimi S, Stilma JS, et al. Retinal detachment in ocular toxoplasmosis. Ophthalmology 2000;107:36-40.

48 Elkins BS, Holland GN, Opremcak EM, et al. Ocular toxoplasmosis misdiagnosed as cytomegalovirus retinopathy in immunocompromised patients. Ophthalmology 1994;101:499-507.

49 Shaikh S, Schwab IR, Morse LS. Association of ocular toxoplasmosis and thymoma. Retina 1997;17:354-6. 